\title{
De nouveaux horizons pour le Journal canadien de la pharmacie hospitalière
}

\author{
par Stephen Shalansky
}

La Société canadienne des pharmaciens d'hôpitaux (SCPH) subit actuellement de nombreux changements, en partie à cause des effets de la pandémie de COVID-19 sur sa situation financière. Le Journal canadien de la pharmacie hospitalière (JCPH) fait partie des initiatives de la SCPH qui visent à atteindre un avenir plus viable. Les récents événements ayant motivé cette réévaluation nous ont également permis de repenser notre approche concernant la publication du JCPH, qui est largement restée inchangée depuis de nombreuses années. Après avoir examiné les dernières données concernant le lectorat, le comité de rédaction a soigneusement examiné les forces et les domaines d'amélioration possibles du Journal ainsi que la manière de tirer profit de l'évolution du paysage de la publication. L'objectif vise à améliorer toutes nos façons de publier le Journal, qui est une ressource de confiance sur laquelle nous nous appuyons depuis de nombreuses années.

Nous y apportons tout d'abord des améliorations technologiques. En ce qui concernela soumission des manuscrits, nous passerons de la plateforme actuelle (eJournalPress) au logiciel Open Journal Systems (OJS) 3. De plus, nous passerons au système OJS 3 pour la publication du Journal. Frais et épuré, l'affichage d'OJS 3 comprend également une interface d'utilisateur fonctionnelle. Elle facilite le processus de soumission, et sa conception dynamique s'adapte à la taille de l'écran des téléphones intelligents, des tablettes et des ordinateurs de bureau. La plateforme permettra en outre de simplifier le flux de travail du personnel du Journal. Vous remarquerez aussi que la présentation du JCPH a été remaniée et que ce format est maintenant plus facile à lire.

Le nombre annuel de numéros du JCPH sera lui aussi réduit et passera de six à quatre. Cette réduction de la fréquence de publication entraînera une légère élévation des critères pour l'envoi de manuscrits à réviser et pour l'acceptation finale. Une autre stratégie visant à préserver la qualité tout en réduisant le nombre de manuscrits publiés annuellement consistera à omettre certaines catégories et à actualiser nos directives en conséquence pour les auteurs. Par conséquent, les types d'articles suivants ne seront plus publiés dans le JCPH : études de stabilité, correspondance (y compris les Communiqués de recherche), critiques de livres ainsi que certains éditoriaux. Nous mettrons également fin à la série Pharmacien praticien avancé. Nous inaugurerons simultanément une nouvelle catégorie qui accueillera des rapports de recherche plus courts (limite de 1500 mots). Cette nouvelle catégorie vise à répondre aux soumissions fréquentes qui ne répondent pas aux exigences relatives à un manuscrit de recherche original de pleine longueur, mais qui comprennent de précieuses informations qui vont au-delà de nos critères relatifs aux Communiqués de recherche. Nous anticipons que de nombreux projets de résidence en pharmacie d'hôpital seront pertinents pour alimenter cette nouvelle catégorie.

Quant aux catégories de manuscrits retenues, nous modifierons la limite du nombre de mots. Par exemple, le résumé des articles des Recherche originales sera limité à 250 mots et le corps de l'article à 3000 mots. Le nombre et la taille des tableaux, leur volume de chiffres et des références seront eux aussi réévalués et réduits, le cas échéant. Nous imposerons aussi une limite de 1500 mots pour la section Innovations en pratique pharmaceutique ainsi qu'une limite de 4000 mots pour les articles Revues. Le Journal se penche également sur la possibilité d'imposer des frais de soumission nominaux (p. ex. $100 \$$ ) pour les manuscrits acceptés à des fins de publication, et cela afin d'améliorer l'engagement des auteurs à l'égard de la qualité de leurs écrits avant de les soumettre. Les directives pour les auteurs du JCPH ont été mises à jour et comprennent maintenant tous les détails de ces changements. Vous les trouverez à https://www.cjhp-online.ca/public/cjhp_directives_aux_ auteurs.pdf

Enfin, le JCPH saisit cette opportunité pour remanier la base de données de ses examinateurs. La qualité des manuscrits acceptés et publiés dans le JCPH dépend fortement des commentaires émis par notre très précieux réseau d'experts. Nous contacterons nos examinateurs actuels pour nous assurer de leur intérêt, mais aussi pour vérifier leurs coordonnées et domaines d'expertise spécifiques. Nous encourageons également les contributeurs et les lecteurs $\mathrm{du} \mathrm{JCPH}$ à se porter volontaires en tant que nouveaux examinateurs ou à en proposer d'autres. Ce sera ici une étape clé pour préserver et améliorer la qualité de notre journal. 
Les changements ne sont pas simples, mais souvent nécessaires pour favoriser l'épanouissement du Journal dans un paysage en mutation. La mise en place complète de ces changements prendra du temps et nous demandera de modifier notre approche habituelle de la soumission des manuscrits ainsi que nos attentes concernant le processus de publication. Nous accuserons probablement un certain retard dans le traitement du nombre considérable de manuscrits déjà soumis ou approuvés pour leur publication. Notre petite équipe responsable des publications et notre comité éditorial ont fort à faire pour s'adapter à ces changements. Nous essaierons de maintenir des délais de traitement similaires à ceux auxquels sont habitués les auteurs, mais les personnes qui soumettent leur manuscrit devront toutefois s'armer de patience au cours des prochains mois. Cependant, une fois que chacun se sera adapté à cette nouvelle approche, nous sommes persuadés qu'il en résultera une version plus solide et de meilleure qualité du Journal canadien de la pharmacie hospitalière. Nous vous remercions sincèrement de votre soutien durant la mise en place de ces processus et nous accordons une grande importance aux commentaires que vous nous communiquerez à ce sujet.

[Traduction par l'éditeur]

\begin{abstract}
Stephen Shalansky, B. Sc. (Pharm), Pharm. D., A.C.P.R., F.C.S.H.P., est coordinateur clinique du Département de pharmacie, Providence Health Care; il est aussi professeur clinique à la Faculté des sciences pharmaceutiques de I'Université de Colombie-Britannique à Vancouver, en Colombie-Britannique. II est également rédacteur en chef du Journal canadien de la pharmacie hospitalière. Conflits d'intérêts : Aucune déclaration.
\end{abstract}

Adresse de correspondance :

Dr Stephen Shalansky

Pharmacy Department

Providence Health Care

1081 Burrard Street

Vancouver BC V6Z 1Y6

Courriel : sshalansky@providencehealth.bc.ca

\section{BESTmis better}

\section{One resource for all types of compounding by pharmacies}

\section{WHAT'S INSIDE?}

- Information for pharmacists, pharmacy technicians, planners, architects, engineers-and others who are involved in decisions or activities that affect compounding

- Guidelines for aseptic compounding, non-aseptic compounding, and compounding which involves hazardous drugs-including radiopharmaceuticals

- Best and leading guidelines on topics such as training, planning and designing the physical environment, developing an air quality strategy, cleaning and decontaminating areas, monitoring the environment, garbing and hand hygiene, developing compounding procedures, documenting, and much more-all in only 230 pages

\section{r}

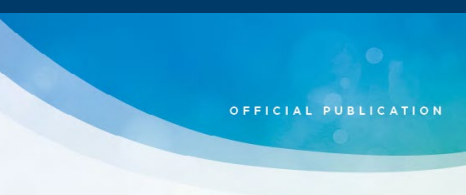

Compounding: Guidelines for Pharmacies

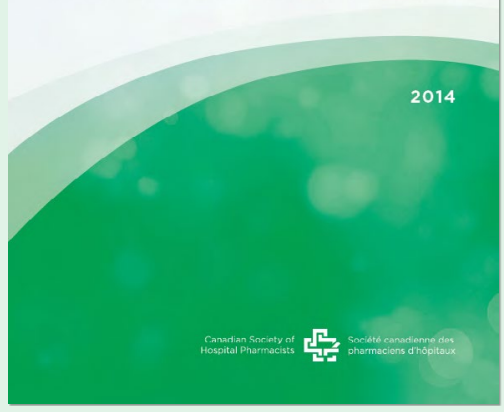

Learn what best looks like: add this publication to your library!
HAVE A SNEAK PEEK OR ORDER AT:

https://www.cshp.ca/compounding-guidelines-pharmacies CSHP MEMBERS PAY A DISCOUNTED PRICE
Canadian Society of Hospital Pharmacists
Société canadienne des pharmaciens d'hôpitaux 